International Journal of Medical Anesthesiology 2020; 3(4): 169-173

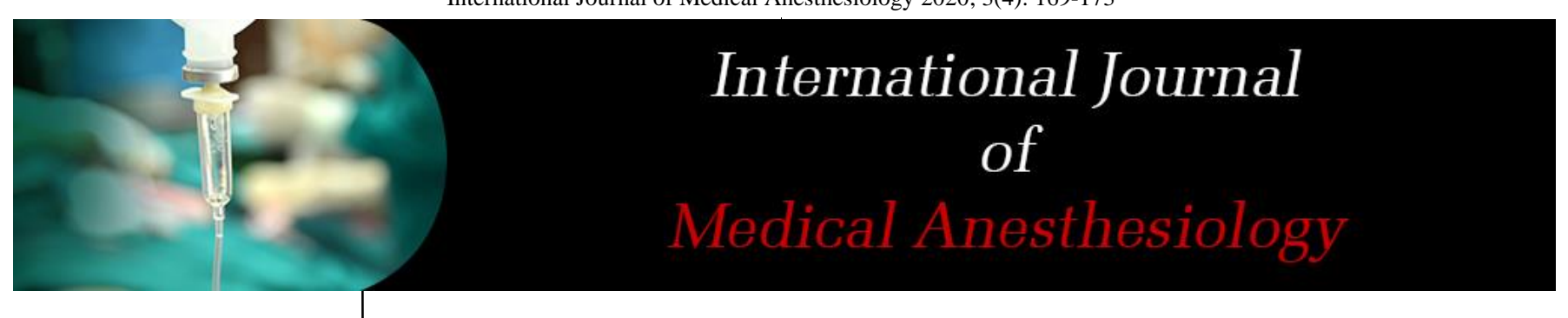

E-ISSN: 2664-3774

P-ISSN: 2664-3766

www.anesthesiologypaper.com

IJMA 2020; 3(4): 169-173

Received: 30-09-2020

Accepted: 24-10-2020

Dr. Sunitha Harish

Assistant Professor,

Department of

Anaesthesiology, Mount Zion

Medical College, Chayalode,

Adoor, Kerala, India
Corresponding Author: Dr. Sunitha Harish Assistant Professor, Department of

Anaesthesiology, Mount Zion Medical College, Chayalode,

Adoor, Kerala, India

\section{Oral clonidine as premedication for attenuation of hemodynamic response during carbon dioxide insufflation in laparoscopic cholecystectomy}

\section{Dr. Sunitha Harish}

DOI: $\underline{\text { https://doi.org/10.33545/26643766.2020.v3.i4c.182 }}$

\begin{abstract}
In this study, we have evaluated the hemodynamic effect of clonidine, administered as a premedication in patients undergoing laparoscopic cholecystectomy. This study reveals that clonidine is an excellent premedication that can suppress the hemodynamic changes associated with induction of anesthesia, during pneumoperitoneum for laparoscopic procedure and in the immediate period after release of the pneumoperitoneum. Clonidine $3 \mathrm{tg} / \mathrm{kg}$, was compared to a placebo $60-90$ minutes before surgery, there were no side effects like bradycardia and hypotension with the use of clonidine. We conclude that clonidine may be an excellent drug to use as a premedication in patients undergoing laparoscopic cholecystectomy.
\end{abstract}

Keywords: Oral clonidine, hemodynamic response, laparoscopic cholecystectomy

\section{Introduction}

Advances in technology and surgical technique have resulted in shorter hospital stay for the patient with consequent reductions in health care costs. The provision of better monitoring facilities, along with increased knowledge and understanding of anatomy and pathology, has allowed the development of endoscopy for diagnostic and operative procedure ${ }^{[1]}$.

Starting in the early 1970s, various gynaecological conditions were diagnosed and treated using laparoscopic procedures. This endoscopic approach was extended to cholecystectomy in the late 1980s. It quickly became apparent that laparoscopy results in multiple benefits compared with open procedures and was characterized by better maintenance of homeostasis. Laparoscopic cholecystectomy is a relatively common procedure, enjoying ever in-creasing popularity and presenting new anaesthetic challenges. The advantages of smaller incision, with less pain and postoperative ileus, with shorter hospital stay has made the procedure more popular than the traditional open cholecystectomy ${ }^{[2]}$. The pneumoperitoneum and the patient positions required for laparoscopy induce pathophysiologic changes that complicate anaesthetic management. An understanding of pathophysiologic consequences of increased intra-abdominal pressure (IAP) is important for the anesthesiologist who must ideally prevent or when prevention is not possible, adequately respond to these changes. The anesthesiologist must evaluate and prepare the patient preoperatively keeping in mind these possible disturbances which can occur intra-operatively [3].

High IAPs (>20 mm Hg) were previously used. After 1990, low 1AP (<15 mm IIg) and modern anesthesia techniques are used. Hemodynamic changes observed during laparoscopy result from the combined effects of pneumoperitoneum, patient position, anesthesia and hypercapnia from the absorbed carbon dioxide. In addition to these pathophysiological changes, reflex increases of vagal tone and arrthymias can also develop. Use of ct2adrenergic agonists such as clonidine or dexmedetomidine and of 13-blocking agents significantly reduces hemodynamic changes and anaesthetic requirements. Use of high doses of remifentanil almost completely prevents the hemodynamic changes ${ }^{[4]}$. Clonidine is one of the drugs that has been extensively studied for attenuating stress response during carbon dioxide insufflation, by administering through oral, intravenous and intrathecal route. The alpha 2 agonist clonidine has shown properties that are potentially beneficial in laparoscopic surgery. It reduces sympathetic activity, postoperative shivering, dries secretions, minimize 
fluctuations in the hemodynamic profile during an-aesthetic induction, and decreases both opioid and volatile anesthetic requirement. Clonidine provides significant benefits in terms of reduced preoperative anxiety and reduced postoperative pain. It is of interest to note the effectiveness of clonidine given orally as a premedication to attenuate the haemodynamic effects of pneumoperitoneum in laparoscopic surgeries. This study was undertaken to evaluate the effect of oral clonidine on hemodynamics, when used as premedication in laparoscopic procedures ${ }^{[5,6]}$.

\section{Methodology}

A prospective randomized single blinded study was conducted at a Tertiary care hospital.

\section{Inclusion criteria}

- ASA I and ASA 11 patients

- Weight 50-70kgs

\section{Exclusion criteria}

ASA III and IV Patients with hypertension, ischemic heart disease, valvular stenotic lesions, left entricular failure and $\mathrm{A}-\mathrm{V}$ conduction blocks. Patients on clonidine. methyldopa, beta blockers and benzodiazepines.

\section{Sample size}

In this study 30 persons were conducted in each group.

- Group A: control group without clonidine.

- Group B: Patients receiving clonidine (3mcgikg) orally $60 \mathrm{~min}$ prior to surgery.

\section{Method premedication}

Oral Alprazolam $0.5 \mathrm{mg}$ on the previous night of surgery.

\section{Procedure}

Oral clonidine given 60minuts before surgery with sips of water at dose $3 \mathrm{mcg} / \mathrm{kg}$ to all patients in group B. Group A did not receive clonidine. Baseline HR, SBP, DBP, MAP, $\mathrm{Sp} 02$ recorded in both the groups

\section{Results}

Table 1: Comparison of age distribution

\begin{tabular}{|c|c|c|c|c|}
\hline \multirow{2}{*}{ Age (Yrs.) } & \multicolumn{2}{|c|}{ Group A } & \multicolumn{2}{c|}{ Group 13 } \\
\cline { 2 - 5 } & No & "A & No & 16.7 \\
\hline $20-30$ & 2 & 6.7 & 2 & 6.7 \\
\hline $31-40$ & 5 & 16.7 & g & 26.7 \\
\hline $41-50$ & 5 & 16.7 & 5 & 7 \\
\hline $51-60$ & 6 & 20.0 & 3 & 23.3 \\
\hline $61-70$ & 7 & 23.3 & 30 & 10.0 \\
\hline$>70$ & 5 & 16.7 & \multicolumn{2}{c|}{$51.03+16.43$} \\
\hline Total & 30 & 100.0 & 700.0 \\
\hline
\end{tabular}

Samples are age matched with $p=0.399$

The above table shows that the age distribution of patients in the two groups were comparable. There was statistically no significant difference between the two groups ( $p$ value$0.399)$.

Table 2: Gender distribution of patients

\begin{tabular}{|c|c|c|c|c|}
\hline \multirow{2}{*}{ Gender } & \multicolumn{2}{|c|}{ Group A } & \multicolumn{2}{c|}{ Group B } \\
\cline { 2 - 5 } & No & \% & No & \% \\
\hline Male & 16 & 53.3 & 13 & 43.3 \\
\hline Female & 14 & 46.7 & 17 & 56.7 \\
\hline
\end{tabular}

Samples are gender matched with $13=0.438$

Both the groups were comparable with respect to sex distribution and there was no statistically significant

Table 3: Comparison of percentage difference in systolic blood pressure (SBP) with baseline values between two groups of patients

\begin{tabular}{|c|c|c|}
\hline SBP & Group-1 & Group-2 \\
\hline L1 & 22.95 & 9.21 \\
\hline L5 & 22.56 & 2.29 \\
\hline P1 & 34.37 & 4.51 \\
\hline P5 & 40.66 & 9.25 \\
\hline P10 & 44.22 & 9.83 \\
\hline P30 & 36.37 & 8.29 \\
\hline AP & 35.96 & 6.86 \\
\hline EXT & 16.79 & 2.37 \\
\hline
\end{tabular}

The percentage difference of systolic blood pressure from baseline (after premedication) in the two group of patients at various time intervals after laryngoscopy and pneumoperitoneum. There was statistically no significant difference in the percentage difference of SBP at 1 minute after laryngoscopy in the two groups of patients as shown in the table above.

The percentage difference of SBP at 5 minutes after laryngoscopy, 1, 5, 10, 2030 minutes after initiation of puemoperitoneum, after release of pneumoperitoneum and 
extubation in the group B (clonidine group) was significantly lower when compared with the group A (no clonidine) patients. The difference between the two group of patients was statistically highly significant as shown by the $p$-value $<0.001$.

Table 4: Mean and standard deviation of percentage difference in SBP (With premedication SBP) at various time intervals in both the groups

\begin{tabular}{|c|c|c|c|c|c|c|c|}
\hline \multicolumn{4}{|c|}{ Group-1 } & \multicolumn{4}{|c|}{ Group-2 } \\
\hline SBP & $\mathrm{n}$ & Mean & SD & $\mathrm{n}$ & Mean & SD & $p$ value \\
\hline $\mathrm{Ll}$ & 30 & 22.96 & 31.02 & 30 & 9.21 & 22.21 & 0.46 \\
\hline L5 & 30 & 22.56 & 27.75 & 30 & 2.29 & 17.26 & $<0.001$ \\
\hline P1 & 29 & 34.37 & 30.29 & 30 & 4.95 & 20.57 & $<0.001$ \\
\hline P5 & 30 & 40.66 & 26.93 & 30 & 9.26 & 23.89 & $<0.001$ \\
\hline P10 & 30 & 44.22 & 28.96 & 30 & 9.83 & 21.27 & $<0.001$ \\
\hline P20 & 30 & 36.37 & 28.35 & 30 & 8.28 & 18.34 & $<0.001$ \\
\hline P30 & 30 & 35.96 & 27.41 & 30 & 6.85 & 19.78 & $<0.001$ \\
\hline $\mathrm{AP}$ & 30 & 16.78 & 24.28 & 30 & 2.37 & 16.84 & $<0.001$ \\
\hline EXT. & 30 & 47.38 & 30.69 & 30 & 20.07 & 26.93 & $<0.001$ \\
\hline
\end{tabular}

Table 5: Comparison of percentage difference in diastolic blood pressure (DBP) from baseline value between two groups of patients

\begin{tabular}{|c|c|c|}
\hline DBP & Group-1 & Group-2 \\
\hline L1 & 18.51 & 16.91 \\
\hline L5 & 17.61 & 8.35 \\
\hline P1 & 24.93 & 16.22 \\
\hline P5 & 24.97 & 21.14 \\
\hline P10 & 27.29 & 17.81 \\
\hline P20 & 23.38 & 19.26 \\
\hline P30 & 20.36 & 16.54 \\
\hline EXT 1 & 7.56 & 15.77 \\
\hline
\end{tabular}

The percentage difference of diastolic blood pressure (with premedication DBP) of patients in the two groups. The percentage difference of DBP at 1,5 minute after laryngoscopy, 1, 5, 10, 20, $30 \mathrm{mins}$ after onset of pneumoperitoneum and at extubation were not significantly different in the two groups as shown by the $p$ values above.

Table 6: Comparing the n, Mean and standard deviation of percentage difference in DBP (From premedication DBP) at various time intervals in both the group

\begin{tabular}{|c|c|c|c|c|c|c|c|}
\hline \multicolumn{9}{|c}{ Group-1 } & \multicolumn{3}{c|}{ Group-2 } \\
\hline DBP & $\mathbf{n}$ & Mean & SD & n & Mean & SD & $p$ value \\
\hline L1 & 30 & 18.52 & 29.88 & 30 & 16.91 & 26.59 & 0.96 \\
\hline L5 & 30 & 17.61 & 27.68 & 30 & 835 & 21.95 & 0.11 \\
\hline PI & 30 & 24.92 & 40.96 & 30 & 16.22 & 26.46 & 0.57 \\
\hline P5 & 30 & 24.58 & 33.03 & 30 & 21.15 & 28.97 & 0,83 \\
\hline P10 & 30 & 27.29 & 34.48 & 30 & 17.81 & 27.27 & 0.35 \\
\hline P20 & 30 & 23.39 & 33.27 & 30 & 19.27 & 24.49 & 0.76 \\
\hline P30 & 30 & 20.36 & 28.31 & 30 & 16.54 & 29.22 & 0.57 \\
\hline AP & 30 & 7.56 & 32.02 & 30 & 15.76 & 28.41 & 0.21 \\
\hline EXT & 30 & 27.04 & 27.04 & 30 & 33.69 & 41.67 & 0.51 \\
\hline
\end{tabular}

Table 7: Comparison of percentage difference of mean arterial pressure from baseline values at various intervals in the two groups of patients

\begin{tabular}{|c|c|c|}
\hline MBP & Group-1 & Group-2 \\
\hline L1 & 19.55 & 13.05 \\
\hline L5 & 20.28 & 5.64 \\
\hline P1 & 29.01 & 12.35 \\
\hline P5 & 32.07 & 17.93 \\
\hline P10 & 33.26 & 15.39 \\
\hline P20 & 30.59 & 15.05 \\
\hline P30 & 28.61 & 13.61 \\
\hline AP & 12.87 & 12.01 \\
\hline EXT & 35.52 & 28.51 \\
\hline
\end{tabular}

\section{Discussion}

Laparoscopic cholecystectomy has revolutionized gall bladder surgeries and it has now become the gold standard for the treatment of cholelithiasis. It offers many benefits over conventional cholecystectomy.

Advantages compared with open cholecystectomy are less pain, smaller incision, better cosmesis, shorter hospitalization, earlier return to full activity and reduced total costs.

However, this procedure is not risk free. In fact it produces significant hemodynamic changes especially in elderly and hemodynamically compromised patients. Pneumoperitoneum affects several homeostatic systems leading to alterations in acid-base bal-ance, cardiovascular, pulmonary physiology and stress response ${ }^{[7]}$.

The extent of cardiovascular changes associated with pneumoperitoneum include an increase in mean arterial pressure, decrease in cardiac output and increase in systemic vascular resistance which in turn compromise tissue perfusion ${ }^{[8]}$. 
Hemodynamic changes observed during laparoscopy result from the combined effects of pneumoperitoneum, patient position, anesthesia, and hypercapnia from the absorbed carbon dioxide. Several mediators had been proposed which included catecholamines, prostaglandins, renin and vasopressin. Unfortunately, no study has correlated hemodynamic changes with changes in levels of these potential mediators.

The increase in systemic vascular resistance can be corrected by the administration of vasodilating anesthetic agents, such as isoflurane or direct vasodilating drugs, such as nitroglycerin or nicardipine. Beta blockers and opioids can also be used to attenuate these hemodynamic responses. The alpha2 agonist clonidine has shown properties that have potential applications as an oral premedication. Number of studies has shown that using oral clonidine premedication helps to attenuate the hemodynamic response to intubation and pneumoperitoneum. The usual practice in our institution, does not include clonidine premedication for patients coming for laproscopic cholecystectomy.

In the present study, 60 patients in two groups of 30 patients each, Group A (Control group) and Group B (Clonidine group) were studied to compare the clinical efficacy of oral clonidine premedication in prevention of hemodynamic response associated with pneumoperitoneum with reference to 1) Heart rate 2) Systolic blood pressure 3) Diastolic blood pressure 4) Mean blood pressure 5) Intra-operative use of Propofol Systolic Blood Pressure. In the present study the hemodynamic changes were compared between patients who received clonidine premedication and control group patients. The percentage difference in $\mathrm{SBP}$ (to the premedication SBP) in the patients who received clonidine premedication was low at 5 minutes after laryngoscopy, 1, $5,10,20,30$,minutes after initiation of pneumoperitoneum, after release of pneumoperitoneum and extubation when compared to the control group patients. This difference was statistically very significant $p$ value $<0.001$.

This result was very similar to the study done by Mrinmoy et al. [9] where patients who received clonidine premedication had lower SBP before induction, after intubation, before pneumoperitoneum, 15, $30 \mathrm{~min}$ after initiation of pneumoperitoneum, after release of pneumoperitoneum and at extubation when compared with patients who received ranitidine as premedication. Similar results were seen in studies done by Toshu Yotsui et al. and Chun-Sung et al. ${ }^{[10]}$. Diastolic Blood Pressure In the present study the percentage difference in diastolic blood pressure (DBP) (with premedication DBP) at 1,5 minute after laryngoscopy, 1, 5, 10, 20, 30 minutes after onset of pneumoperitoneum, after release of pneumoperitoneum and at extubation were not significantly different in the two groups of patients as shown by the p values. These results of our study was comparable with the results of the study by Mrinmoy et al. were the DBP before premedication, before initiation of pneumoperitoneum and after release of pneumoperitoneum were not significantly different but the patients in the clonidine group had lower DBP before induction, after intubation, after pneumoperito-neum and after Extubation when compared to the ranitidine group. Similar results were seen in studies done by Toshu Yotsui et al. and Chun-Sung et al. The mechanisms of these actions may involve inhibition of sympathetic outflow and the potentiation of parasympathetic nervous activity. Mean Arterial Pressure The percentage difference of mean arterial pressure (MAP) (with premedication MAP) in the two group at $1 \mathrm{~min}$ after intubation, 5, 20, 30 minutes after pneumoperitonium, after release of pneumoperitoneum and after extubation were not significantly different. The percentage difference of MAP (with the premedication MAP) at 5minutes after laryngoscopy, 1, 10 minutes after initiation of pneumoperitoneum and extubation in the clonidine group was significantly lower when compared with the control group of patients.

Yuvesh Passi et al. in their study had found the MAP to be lower in the clonidine premedicated patients. The results of our study were similar to the study done by Chun-Sung et al. peri-operative MAP was lower in patients of clonidine group than in those of placebo group in their study. Similar results were seen in the study done by Mrinmoy et al. The patients in the clonidine group had lower MAP before induction, after intubation, after pneumoperito-neum, after release of pneumoperitoneum and after extubation when compared to the ranitidine group.

The percentage difference in heart rate (with premedication heart rate) at $1,5,10,20, \mathrm{~min}$ after initiation of pneumoperitoneum and after extubation were very significantly lower in clonidine group of patients when compared to control group patients. While at other time intervals of the study there was statistically no significant difference between the two groups. These findings were similar to the findings in the following studies.

Sung CS et al. ${ }^{[10]}$ found the pre-induction heart rate was slower in the clonidine group than in the placebo group. The perioperative heart rate which was variable at each time interval was also slower in the clonidine group in comparison with the placebo group. Islam A et al. ${ }^{[22]}$ found in their study the mean difference of pulse rate at different 4 times was significant $(p<0.05)$, however just before induction, just after skin incision and just after insufflations carbon dioxide were not significant $(p>0.05)$.

\section{Conclusion}

Premedication with oral clonidine one hour prior to laparoscopic cholecystectomy is useful in prevention of hemodynamic response associated with pneumoperitoneum. Clonidine also decreases the requirement of propofol intraoperatively during pneumoperitoneum. Hence, routine premedication of oral clonidine $(1501 / \mathrm{g})$ one hour before laparoscopic cholecystectomy is recommended.

\section{References}

1. Miller RD. Miller textbook of anaesthesia. In. Anesthesia for Laparoscopic Surgery. Joris JL. 7th edition: Elsevier Churchill Livingstone 2010, P21852197.

2. Cummingham AJ, Brull Si. Laparoscopic cholecystectomy: anesthetic implications. Anesth Ana 12 1993;76:1120-33.

3. Hayashi Y, Maze M. Alpha-2 adrenoceptor agonists and anaesthesia. Br J Anesth 1993;71:108-118.

4. Skandalaki JE. Skandalaki's Surgical Anatomy. In. Extrahepatic biliary tract and gallbladder. Karaliotas CC et al. Paschalidis Medical Publications 2004, P1117-1130.

5. Litynski GS. Profiles in laparoscopy: Mouret, Dubois,and Perissat: The laparo-scopic breakthrough in Europe (1987-1988) ISLS 1999;3:165-167.

6. Jarnagin WR. Blumgart Surgery of liver. In. Biliary 
tract and pancreas, $4^{\text {th }}$ Edition 2 P506-522.

7. Cummingham AJ, Cathal Nolan. Anesthesia for minimally invasive procedure. In Clinical Anesthesia, 5th Edition P2207.

8. Stocking RK. Pharmacology and physiology in anaesthesia practice. In. Antihyper-tensive drugs. 31-d edition. Lippincott-Raven Publishers 1999, P304-307.

9. Malek KJ, Knor J. Kurzova A. Adverse haemodynamic changes during laparoscopic cholecystectomy and their possible suppression with clonidine premedication. Comparison with intravenous and intramuscular premedication, Rozhl chir 1999:78:286-291.

10. Sung CS, Lin SH, Chan KH. Effect of oral clonidine premedication on perioperative haemodynamic response and postoperative analgesic requirements for patients undergoing laparoscopic cholecystectomy. Acta Anaesthesiol Scand 2000;38:23-29. 